\title{
Switching factor products: selecting patients and managing the process
}

Christine Harrington*, Charles Hay, Vicky Vidler, Rashesh Dattani, Katherine Heygate

Clotting factor concentrates used in the treatment of haemophilia are now procured centrally in the UK by nationally organised competitive tendering to obtain the best product price while still preserving some degree of prescribing freedom. Inevitably, however, some patients are required to switch from one brand of factor VIII to another to fulfil contractual requirements. This process of switching patients from one product to another falls to staff at individual haemophilia centres, with haemophilia nurses in particular playing a key role. This article outlines the national procurement process and discusses how the switch can be managed quickly and smoothly, as well as providing practical guidance on selecting which patients may be asked to switch. Issues such as patient communication, managing potential patient anxiety and efficient stock management are also discussed.

Key words: haemophilia, national tender, switching, factor VIII

The widespread adoption of prophylaxis and subsequent increased spend on clotting factor concentrates over the past decade has resulted in haemophilia becoming one of the most expensive conditions to treat. The UK used 475 million units of factor VIII in 2011, and in 2011/12 the average adult with severe haemophilia $A$ in the UK used 250,000 units, at a cost in excess of $€ 100,000$ per year [1]. Until 2005 each haemophilia centre purchased factor VIII and IX directly from the manufacturers, often using an informal procurement process which frequently failed to yield the best price. These rapidly growing costs led to the decision to procure clotting factors centrally through competitive tendering.

National competitive procurement now operates in a number of countries, and in the UK this tender process has achieved considerable cost reductions while retaining all suppliers and maintaining a high level of involvement from clinicians, as well as a degree of prescribing freedom [1].

The tender process is only the start of the procedure, however, and it is then up to individual haemophilia

\footnotetext{
* Christine Harrington, Nurse Consultant (Haemophilia), Katharine Dormandy Haemophilia Centre and Thrombosis Unit, Royal Free Hospital, London NW3 2QG. Email: c.harringtonanhs.net

Charles RM Hay, Professor of Haemostasis and Thrombosis, Consultant Haematologist and Director, Manchester Haemophilia Comprehensive Care Centre, Manchester Royal Infirmary

Vicky Vidler, Nurse Consultant (Paediatric Haematology), Sheffield Children's Foundation NHS Trust

Rashesh Dattani, Clinical Nurse Specialist/Centre Manager, Haemostasis and Thrombosis Centre, Leicester Royal Infirmary

Katherine Heygate, Senior Medical Advisor, Novo Nordisk UK
}

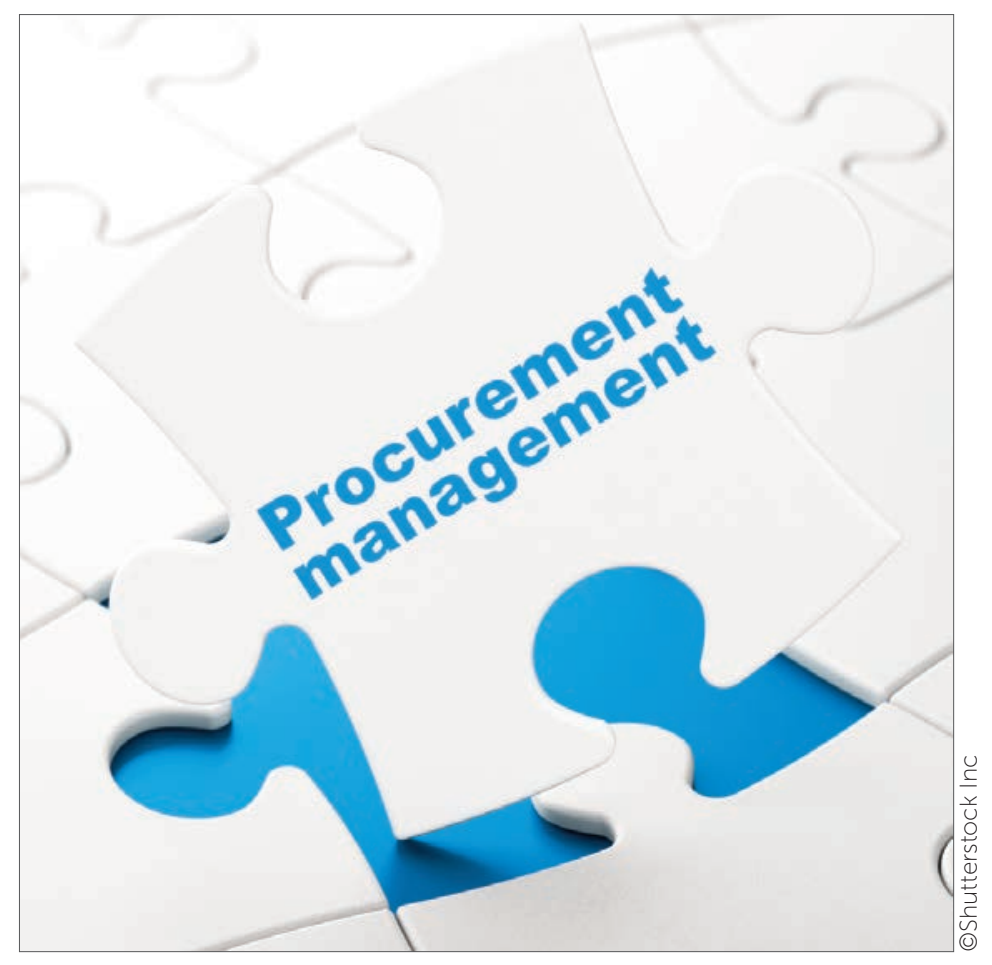

centres to organise and implement the switchover. Staff at the centres are the key to a swift and smooth operation and preparation is crucial.

\section{The procurement process}

The central procurement of recombinant clotting factor products in the UK by the Department of Health now means that instead of each haemophilia centre purchasing its own supply directly from the manufacturers, the government procures sufficient for the entire country using a national tender system. Buying large volumes and obtaining competitive bids from manufacturers achieves cost savings by guaranteeing that the NHS pays the best price for recombinant clotting factors.

In previous UK tenders, each product was scored on its safety, efficacy, convenience of use, security of supply and unit cost. The products were then ranked according to the points scored and those with the highest scores were awarded the larger shares of the market. The market share ranged from $10-40 \%$ of the total volume (for more details on the tender process see [1]).

While this method of procurement is extremely cost effective, reducing the unit prices of recombinant factor VIII by almost half in six years, it also required almost 50\% of patients to switch brand in order to achieve centrally 


\section{Clinical leadership key to UK tendering}

Charles Hay, Director of the Manchester Haemophilia Comprehensive Care Centre at Manchester Royal Infirmary, outlines the UK tender process

Companies that wish to supply clotting factor products in the UK are asked to submit a tender supported by product and manufacturing data. Each tendered product is subject to a structured multidisciplinary technical evaluation based on its safety, efficacy, convenience of use, security of supply, and price.

For the 2006 national contract, technical evaluation formed $60 \%$ of the final score and price made up $40 \%$. However, technical evaluation did not differentiate very much as products were so similar in efficacy and safety. For the most recent contract round, the process was based on a ranking of products with $25 \%$ based on technical evaluation and $75 \%$ on unit price, an approach that encourages manufacturers to behave competitively.

Efficacy and safety were evaluated by clinicians; security of supply was evaluated by clinicians and commissioners. Ease of use was evaluated by nurses and patients using sample products and infusion sets. The technical evaluation was broken down by efficacy, safety, convenience, and security of supply. Commitment to the clinical area (so-called 'added value') such as support and education courses for nurses was not included in the evaluation since the commissioners did not consider these to be valuable activities, and they could only be evaluated subjectively

No supplier was excluded from the UK market even though this strategy has a financial cost. It was considered important to keep all suppliers in the market to maximise security of supply and to maintain clinicians' prescribing freedom, while also ensuring access to pipeline products.

The final tender price was established through a reverse e-auction. The final award was decided at an adjudication meeting at which clinicians and specialist commissioners assessed the technical evaluation and final tender price and, assisted by simultaneous computer modelling, decided what proportion of the market to award to each supplier. The supplier with the highest product ranking was awarded $40 \%$ of the market, second $25-40 \%$, third $25 \%$ and fourth $10 \%$.

Each region is expected to purchase and prescribe in compliance with the proportions of products awarded in the contract. There is enough flexibility for smaller centres to be able to choose to prescribe a single product for administrative ease, with neighbouring large centres generally 'taking up the slack' and prescribing in such a way that the region complies with the contract.

Although complex, the UK approach to clotting factor procurement has successfully reduced unit cost while preserving some degree of clinical control and prescribing freedom. The success of this process was in no small measure attributable to the high level of clinician involvement, clinician commitment to the process and clinical leadership. There is far less medical input in other countries with national procurement, such as Canada, New Zealand and Australia. agreed contract volumes awarded to each manufacturer.

Centres take a multidisciplinary approach when deciding which patients to switch, however, the responsibility for managing the resource-intensive and time-consuming process often falls to haemophilia nurses. There is no national guidance for the switching of patients and it is important for nurses to be well prepared in advance of the switch so they can move swiftly to identify which patients to switch and which patients should remain on the same product.

It is also important to address home and centre stock management so that the centres, and patients who receive home delivery, are not left with large quantities of products that have to be used before the centre can manage the proportions allocated under the new contract. Home and centre stock of products being phased out have to be run down in anticipation of the start of the new contract.

\section{Choosing which patients to switch products}

Before identifying suitable patients to switch products each patient must first be considered on both clinical and personal levels to enable any transition to a different product to progress smoothly.

Preplanning is therefore essential and in preparation for the tender in April 2014 centres were advised to be regularly monitoring usage in their centres and the volumes of each product used. Once the outcome of the tender was known, nurses then needed to be prepared to manage the switching process quickly, although previous experience has shown that it can take up to 3 months to switch all patients.

The choice of treatment product for patients with haemophilia has been a sensitive issue since the era of transfusion-transmitted pathogens in the 1970s and 1980s. Although this risk was addressed, first with the viral inactivation of plasma-derived products and then the introduction of recombinant factor concentrates, some patients can feel vulnerable if they have to switch products, particularly if there is a family history of contracting a blood-borne virus.

Choosing who to switch ideally involves a tailored and highly personalised approach, which draws on the haemophilia team's knowledge of patients and their families. The outcome of the last national contract evaluation was that there is no evidence to differentiate between recombinant factor VIII products in terms of efficacy or safety and also that there is no evidence of an increased risk of developing inhibitors linked specifically to changing product $[2,3,4]$.

It is important, however, to consider recognised and 


\section{The paediatric perspective}

Vicky Vidler, Nurse Consultant (Paediatric Haematology) at Sheffield Children's NHS Foundation Trust, outlines handling the switch in paediatric patients

The most important aspect when switching clotting factor concentrates for children is for the centre to have a good and open relationship with patients and their parents and to be available to talk through any concerns or queries they may have. In Sheffield, we wrote to all the parents of children we proposed switching, explaining the rationale for the changes, including how switching saves the NHS money. We also explained that all recombinant products are broadly similar and that their safety profiles are all good. Families were invited to phone us or come in and see us if they had any questions, but none of them chose to do so.

Many of the children at our centre are the first in their families to have haemophilia and most have only ever been treated with recombinant factor VIII. It is a testament to the treatment advances over recent years that the paediatric haemophilia community does not have the same degree of anxiety associated with past treatments. However, they do still want to understand about current clotting factor concentrates and future developments.

After the first tender process the centre did not have to change anybody, but after the second tender quite a number of children were switched. They have been regularly checked since for inhibitors and none have developed. This is an important consideration that would influence our decision making in the future. If past inhibitor patients have to be changed it would be important to take into consideration how long ago they had an inhibitor and how difficult it had been to treat and eradicate. If possible, brothers and those living in the same household should be kept on the same product.

It was relatively easy to select which children to switch as all of those we changed were on the same concentrate and none of them had previously had an inhibitor. We took into consideration the number of exposure days each child had, as we know that the risk of inhibitor formation is highest during the early exposures to clotting factor concentrates. The median time to inhibitor presentation is $10-15$

exposures to treatment [5].

Certain practical aspects should also be taken into consideration, e.g. to make sure that the user kit and ancillary items are easy to use. Boys get quite a sense of security out of following the same treatment routine and it may take time for them to feel comfortable with any changes. These issues can be acknowledged and addressed during routine clinic reviews in the first few months after switching products.

All our patients are on home delivery and a great deal of pre-planning went into ensuring that the home delivery company was aware of our plans and timescales. Effective communication with them was very important and it was crucial to make sure all the prescriptions for the new products were ready quickly once we knew the results of the tender process.

Switching patients is very much a team effort. Although time consuming, with the right planning and the support and co-operation of patients and parents the process can be successfully completed and thereby realise significant savings for the NHS. potential risk factors for inhibitor formation before deciding which patients to switch. These are well described in the literature, with current UKHCDO guidelines on the management of inhibitors [5] listing risk factors in previously untreated patients as:

- Genetic mutation

- Ethnicity

- Family history [6]

- Age - risk is highest below the age of five years and increases after the age of 60 years

- HIV status

- Treatment-related previous exposure days (EDs): risk is highest during early exposures with a median time of inhibitor presentation at about 10-15 EDs [7,8]. Risk is lower after 150 EDs but may occur throughout life [9]. - Intense exposure: risk is increased with five or more EDs at first treatment (severe haemophilia A) [10]. Risk is increased with intense exposure (mild haemophilia A) - Surgery: centres would be unlikely to switch the factor product of a patient scheduled for surgery because of the increased risk of inhibitors.

Other than increasing age, none of these risk-factors have been shown to apply to the previously treated patients who make up the vast majority of patients being considered for a product-switch. There are many other aspects to consider, which are often dependent on the haemophilia teams' detailed knowledge of patients and families.

\section{First steps to change}

The first step is to examine the centre's data on the use of recombinant factor VIII products and to compare the total proportions of different products with the totals allocated under the national contract. This will identify the number of units per year that will need to be allocated to a different product. It is important to:

- Take into account the number of units used per year by each individual patient and their pattern of use. It is notable that in the recent switch, centres tended to switch patients who used more factor VIII, thus minimising the number of patients required to switch products to achieve their contracted factor VIII volume targets

- Examine those with high use and determine how many of these patients would need to switch to meet the national tender allocation

- Consider how many lower users would have to be 


\section{The key to successful switching}

Rashesh Dattani, Clinical Nurse Specialist at the Haemostasis Thrombosis Unit, Leicester Royal Infirmary, discusses how to manage the switching process

The key to a smooth and successful switch in both adult and paediatric patients is planning, preparation, and patient confidence. Most patients and parents are aware of the cost of the therapies and the basis of the tendering process and why it has to be done. Most important is how the switch is explained to them. In Leicester, we do not say patients are switching to a cheaper product as they invariably assume this means it is inferior. Instead we explain that it is a product we can get for a better price.

Preparation and planning begins 2-3 months in advance of the tender. We identify patients who might have to change and when they come to clinics the possibility is mentioned to them (around January/February for an April tender). Patients are warned that they may have to change products. We have to obtain patients' consent and ensure they are happy.

We have a very close relationship with our adult patients and also with parents and children. They know they can pick up the phone and ask questions at any time. They also know we have their best interests at heart. If they have confidence in you and your team most patients will accept your advice.

There is no hard and fast rule as to who to change. Each patient is considered individually, meaning it is time consuming. In our clinic we tend to change the higher users. We also try to keep families on the same product.
Most of our patients are very relaxed and as long as the product is working they are happy and accepting of change when it happens. Some patients do not want to move at all although we have never had anybody refuse.

Fear of inhibitors is the main patient (and parent) concern. They also want to know why there is a need to change products if they have not had any bleeding. If there is a theoretical risk of inhibitors (e.g. due to a known predisposing genetic mutation) this is taken into account. If patients are known to have had inhibitors in the past we try to keep them on the same treatment irrespective of tendering. There can also be a genetic connection to inhibitors so if there is a family history of inhibitors we will not change that particular patient. For example if a young child has never had an inhibitor but his grandfather has there could be an inherited risk. We are fortunate that in the past 15-20 years risk of inhibitors in our centre has been low, with only one child who had inhibitors since birth.

Some patients are also on plasma products. The donor pool of these is very large (approximately 20,000) and therefore to minimise the risk of exposure to unknown prions we ask for same batch number delivery. Although with recombinant products the risk of infection is minimal/theoretical we still work on the same principle as for plasma products and try not to mix batch numbers. Some patients are notoriously poor at recording the batch numbers of the treatment they give themselves so it also makes easier for the centre to keep records of batch numbers delivered by the companies. Also, in the past, on the occasions when mixed batch numbers have been delivered to patients they have not separated the batches. This has complicated reporting to the national database and subsequently makes finding out batch number exposure time-consuming for staff.

It is also important to start reducing stock levels 2-3 months before the tender so that leftover stock is minimal. We work closely with home delivery companies to reduce their stocks as well as any stock that patients may have at home. For this reason home treatment records are important so we know how much factor patients are using and how many bleeds they have. On average patients have deliveries every 2 months, and hold 8 weeks stock and 2 weeks' buffer stock to cover bleeds. Once the tender is announced we have 2 months to implement the switch and on the last occasion we were able to change most of our patients in that time. They were able to use up what factor they had and leftover stock was minimal.

When we switch patients to a different product we carry out 24-hour trough level profiling. Each patient has his own individual half-life profile, and so these studies allow the centre to advise the patient appropriately as to how much treatment they require for joint bleeds, muscle bleeds etc, and when to treat again, i.e. 6, 8, 12 or 24 hours later. We check for inhibitors before changing and continue to check at regular intervals for up to 3 months to ensure patients are not developing inhibitors. If they are getting more bleeds on the new product it may either be an early sign of an inhibitor or it could be due to increased physical activity of the patient. switched - it may be necessary to consider what the approach will be if some patients choose not to change product

- Draw up a list of those who might potentially be switched

- Review list with centre colleagues.

Additional consideration and thought should be given to paediatric patients, especially very young children who have only received a low number of factor VIII infusions. It is sensible and convenient for all members of a household to be on the same clotting factor concentrate.

\section{Products - generations and safety}

Products are categorised as first, second or thirdgeneration depending on when they were licensed and whether they contain human and/or animal proteins. First generation products (no longer used in the UK) contained human albumin (as a stabiliser) and animal plasma Second-generation products contain no human albumin but use animal products such as foetal calf serum to incubate the mammalian cell line used in the manufacturing process. Third-generation products contain no human or animal proteins in their manufacture. 


\section{SWITCHING CHECKLIST}

Monitor factor usage/volumes of each product used

Examine data on use for each product

up list of who might potentially be switched

Write to patients regarding switch

- Units used per year per patient

- Determine high users

- Determine low users

- Follow up with phone call

- Invite patient to discuss switch over the phone or in person

- Run down home therapy stock and manage hospital stock in advance of the switch so that switching can take place as soon as possible after the switch date

Check for inhibitor before switching

After switch, monitor for bleeds and/or inhibitors

Following previous tenders it has not been necessary to switch patients on third-generation products to secondgeneration products. In order to meet the stipulated volumes, patients on second-generation products were switched to other products of the same generation.

It should be borne in mind that there may be a perception on the part of some patients that more recent generation concentrates are safer than their predecessors. A recent systematic review of prospective studies published in the last 20 years undertaken on behalf of the European Haemophilia Safety Surveillance (EUHASS) study group found no differences in the rate of adverse events caused by plasma-derived or recombinant factor products [11].

Most patients do not differentiate between recombinant factor VIII concentrates and are just concerned to remain on a recombinant product. Some have a more complex view on the safety of the different factor products, which may in part reflect the way healthcare professionals have explained the differences between the generations of product in the past.

In addition, some patients may be reluctant to change products because they perceive there to be a greater inhibitor risk associated with different products or when switching between products, particularly in previously untreated patients (PUPS). A recent analysis of data from 574 patients with severe haemophilia A PUPs concluded that recombinant and plasma-derived factor VIII products conferred similar risks of inhibitor development. However, an unexpected finding from this study was that secondgeneration full-length recombinant products were associated with an increased risk of inhibitor development, compared to third-generation products [12].

This finding has not been confirmed, however, and there is no evidence to suggest any difference between different factor VIII products in previously treated patients. A recent systematic review and meta-analysis of 28 studies involving 1,421 patients with haemophilia A showed that the different types of FVIII products are not associated with different risks of inhibitor development [11]

It may be the case that if the patient has a history of developing an inhibitor then the clinician might be more reluctant to make any product change. It may be that some patients who have developed a strong sense of trust in the product they currently use would be unduly anxious or uncomfortable about changing.

Some patients may find it more difficult than others to learn how to use a new reconstitution device and it would not be advisable to switch these patients. Also, packaging and infusion kits may be a particular issue for patients with reduced dexterity or impaired coordination, e.g. older patients with joint disease

Some patients and families express strong personal preferences for particular accessory kits and devices. If more than one member of the household is on recombinant factor VIII, consideration should be given to keeping them all on the same product for convenience and stock management reasons. It may be that safety is a particular fear for the patient/family, perhaps because members of the family have been affected by viral transmission in the past.

\section{Communicating with patients}

When communicating the proposed change of product to patients it is best to keep things as simple as possible. Talk to patients openly, explaining the evaluation process undertaken as part of the national tender and providing information about the product proposed for them, why they could switch and maybe why other patients cannot. It is essential to involve the patient in the decision-making process. It is not the nurse's job to persuade patients to switch - any change must be undertaken with the patient's informed consent. Patients must be allowed to opt out but it should be remembered that if one person chooses not to switch product then another must be identified who is willing to change so that the agreed quotas are realised.

As the tender process and consequent product switch has happened twice before, many patients will already be aware of the process. Others will have heard about it from the Haemophilia Society or the haemophilia group on Facebook. In the past, there was little resistance to switching from patients since most have a very low level of concern regarding product switching. Over the years, older patients will have switched products many times for a variety of reasons including product shortages and 
product improvements.

Patients should be written to initially and followed-up with a phone call to invite them to discuss the switch, either in person or over the phone. When discussing the switch with patients it is useful to have demonstration devices to use in training, along with educational materials about the product, the reconstitution device and the infusion kit. It is important to consider that older patients may need extra support. Patient support materials are usually readily available from the manufacturer, and these can be supplied in advance of your patient consultations.

\section{Monitoring}

Advice from the UKHCDO for the implementation of the 2014 contract for patients switching to an alternative factor VIII has been amended from previous guidance. It is now recommended that patients are screened for an inhibitor prior to switching products and within 6 months of treatment with an alternative concentrate. Tests to detect the presence of an inhibitor should also, ideally, be done after a washout long enough to ensure that the baseline factor level has been reached. Although there is a concern that switching products may increase the risk of inhibitor development, current evidence suggests that inhibitor risk is unaffected by switching.

\section{Stock management}

It is important to manage stock and home delivery in advance as once the tender pronouncement is made certain products may no longer be used. Running down the supplies in the home and hospital setting, in conjunction with early liaison with home delivery companies, can minimise the risk of waste when the switch is made. There are also likely to be changes to the volumes, depending on the brand of factor, and therefore more storage space could be required.

\section{Conclusion}

Past experience has shown how to optimise the process of managing the switch of recombinant factor VIII products post tender both for the benefit of patients and haemophilia centres. It is a time-consuming and intensive process but it can be successfully achieved by being proactive and prepared.

Looking to the future, it is important to recognise that while the cost savings for the NHS are significant, this national process may make the use of newer and more costly products harder to justify and adopt. Increased patient and parent participation and involvement should be explored for future contracts. The tender process completed in April 2014 will provide further insights and experience for future treatment changes to be implemented smoothly and efficiently.

\section{Acknowledgement}

This paper is based on a roundtable discussion meeting funded by Novo Nordisk. The authors wish to thank: Jemma Efford, Clinical Nurse Specialist, Great Ormond Street Hospital, London; Emma Franklin, Haemophilia Clinical Nurse Specialist, Bristol Haemophilia Comprehensive Care Centre; Lisa Grainger, Advanced Nurse Practitioner, Oxford Haemophilia \& Thrombosis Centre, Churchill Hospital, Oxford; Kingsley Lawrence, Specialist Haemophilia Nurse, Cambridge Haemophilia Centre, Addenbrooke's Hospital, Cambridge; Barbara Subel, Clinical Nurse Specialist, Haemophilia, Katharine Dormandy Haemophilia Centre \& Thrombosis Unit, Royal Free Hospital, London; Julie Vowles, Nurse Specialist, Haemophilia Centre, Royal Victoria Infirmary, Newcastle Upon Tyne.

Editorial support for production of this paper was provided by Jane Smalls, Meritus Communications, Kent and funded by Novo Nordisk.

\section{References}

1. Hay CRM. Purchasing factor concentrates in the 21st century through competitive tendering. Haemophilia 2013; 19(5):660-7.

2. Sharrer I. Ehrlich $\mathrm{HJ}$. Lack of evidence for increased inhibitor incidence in patients switched from plasma-derived to recombinant factor VIII. Haemophilia 2001; 7: 766. 3. Giles AR, Rivard GE, Teitel J, Walker I. Surveillance for factor VIII inhibitor development in the Canadian Hemophilia A population following widespread introduction of recombinant factor VIII replacement therapy. Transfusion Science 1998; 19(2): 139-148.

4. Rubinger M, Lillicrap D, Rivard GE, et al. A prospective surveillance study of factor VIII inhibitor development in the Canadian hemophilia A population following the switch to a recombinant factor VIII product formulated with sucrose. Haemophitia 2008; 14(2): 281-286

5. Collins P, Chalmers E, Hart DP et al. Diagnosis and treatment of factor VIII and IX inhibitors in congenital haemophilia: (4th edition). UK Haemophilia Centre Doctors Organisation. Haematol 2013; 160: 153-170.

6. Hay CR, Palmer B, Chalmers E, et al. Incidence of factor VIII inhibitors throughout life in severe hemophilia A in the United Kingdom. Blood 2011; 117: 6367-6370. 7. Lusher JM, Arkin S, Abildgaard CF, Schwartz RS. Recombinant factor VIII for the treatment of previously untreated patients with hemophilia A. Safety, efficacy, and development of inhibitors. Kogenate Previously Untreated Patient Study Group. N Engl J Med 1993; 328: 453-459.

8. Lusher JM, Lee CA, Kessler CM, Bedrosian CL. ReFacto Phase 3 Study Group. The safety and efficacy of B-domain deleted recombinant factor VIII concentrate in patients with severe haemophilia A. Haemophilia 2003; 9: 38-49.

9. Rothschild C, Laurian Y, Satre EP et al. French previously untreated patients with severe haemophilia A after exposure to recombinant factor VIII: incidence of inhibitor and evaluation of immune tolerance. Thrombosis and Haemostasis 1998; 80: $779-783$

10. Gouw SC, van den Berg HM, le Cessie S et al. Treatment characteristics and the risk of inhibitor development: a multicenter cohort study among previously untreated patients with severe hemophilia A. Journal of Thrombosis and Haemostasis 2007; 5: 1383-1390.

11. Franchini M, Makris M, Santagostino E, et al. Non-thrombotic-, non-inhibitorassociated adverse reaction to coagulation factor concentrates for treatment of patients with haemophilia and von Willebrand's disease: a systematic review of prospective studies. Haemophilia 2012; 18(3): e164-72.

12. Gouw SC, van der Bom JG, Ljung R et al for the PedNet and RODIN Study Group. Factor VIII products and inhibitor development in severe haemophilia A. N Engl J Med 2013; 368: 231-9.

\section{How was it for you? \\ Are you a nurse, physiotherapist or allied health care professional with experience of the impact of product switching on patients? Share your experiences on Haemnet}

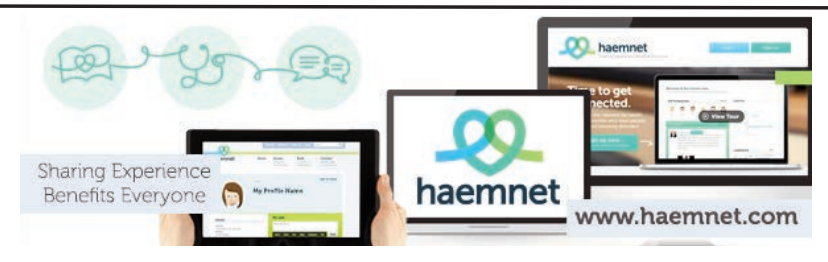

